\title{
An Estimate of the Rate of Convergence of the Fourier Series in the Generalized Hölder Metric by Delayed Arithmetic Mean
}

\author{
L. Nayak, ${ }^{1}$ G. Das, ${ }^{2}$ and B. K. Ray ${ }^{3}$ \\ ${ }^{1}$ School of Applied Sciences, KIIT University, Bhubaneswar, Odisha 751024, India \\ ${ }^{2}$ Department of Mathematics, Utkal University, Vani Vihar, Bhubaneswar 751004, India \\ ${ }^{3}$ Department of Mathematics, Ravenshaw University, Cuttack 751007, India
}

Correspondence should be addressed to L. Nayak; laxmipriyamath@gmail.com

Received 6 December 2013; Revised 16 April 2014; Accepted 16 April 2014; Published 7 May 2014

Academic Editor: Baruch Cahlon

Copyright (C) 2014 L. Nayak et al. This is an open access article distributed under the Creative Commons Attribution License, which permits unrestricted use, distribution, and reproduction in any medium, provided the original work is properly cited.

We study the rate of convergence problem of the Fourier series by Delayed Arithmetic Mean in the generalized Hölder metric $\left(H_{p}^{(w)}\right)$ space which was earlier introduced by Das, Nath, and Ray and obtain a sharper estimate of Jackson's order.

\section{Definition}

Let $f$ be a $2 \pi$-periodic function such that $f \in L_{p}[0,2 \pi], p \geq$ 1. Let the Fourier series of $f$ at $t=x$ be given by

$$
\frac{1}{2} a_{0}+\sum_{n=1}^{\infty}\left(a_{n} \cos n x+b_{n} \sin n x\right)
$$

Let

$$
\phi_{x}(t)=\frac{1}{2}\{f(x+t)+f(x-t)-2 f(x)\} .
$$

Let $S_{n}(x)$ be the $n$th partial sum of (1). Then it is known ([1], page 50) that

$$
S_{n}(x)-f(x)=\frac{2}{\pi} \int_{0}^{\pi} \phi_{x}(t) D_{n}(t) d t
$$

where $D_{n}(t)=\sin (n+1 / 2) t / 2 \sin (t / 2)$ is known as Dirichlet's kernel.

Let $\mathscr{C}_{2 \pi}$ denote the Banach space of all $2 \pi$-periodic continuous functions defined on $[-\pi, \pi]$ under the supremum norm. The space $L_{p}[0,2 \pi]$ with $p=\infty$ reduces to $\mathscr{C}_{2 \pi}$ defined over $[0,2 \pi]$. We write

$$
\begin{aligned}
\|f\|_{c}= & \sup _{0 \leq t \leq 2 \pi}|f(t)|, \quad(p=\infty) \\
\|f\|_{p}= & \left(\int_{0}^{2 \pi}|f(t)|^{p} d t\right)^{1 / p}, \quad p \geq 1 \\
\omega(\delta, f)= & \sup _{0 \leq|h| \leq \delta}\|f(\cdot+h)-f(\cdot)\|_{\mathcal{c}}, \quad f \in \mathscr{C}_{2 \pi} \\
& \omega_{p}(\delta, f)=\sup _{0 \leq|h| \leq \delta}\|f(\cdot+h)-f(\cdot)\|_{p}, \\
& f \in L_{p}[0,2 \pi], \quad p \geq 1,
\end{aligned}
$$

when the norm has been taken with respect to $x$ throughout the paper. The quantities $\omega(\delta, f)$ and $\omega_{p}(\delta, f)$ are, respectively, called the modulus of continuity and integral modulus of continuity of $f$. It is known ([1], page 45$)$ that $\omega(\delta, f)$ and $\omega_{p}(\delta, f)$ both tend to zero as $\delta \rightarrow 0$.

It was Prössdorf [2] who first studied the degree of approximation problems of the Fourier series in $H_{\alpha}(0<$ $\alpha \leq 1)$ space in the Hölder metric. Generalizing the Hölder metric, Leindler [3] introduced the space $H^{\omega}$ given by

$$
H^{\omega}=\left\{f \in \mathscr{C}_{2 \pi}: \omega(\delta, f)=O(\omega(\delta))\right\},
$$


where $\omega$ is a modulus of continuity; that is, $\omega$ is a positive nondecreasing continuous function on $[0,2 \pi]$ with the following property:

(i) $\omega(0)=0$,

(ii) $\omega\left(\delta_{1}+\delta_{2}\right) \leq \omega\left(\delta_{1}\right)+\omega\left(\delta_{2}\right)$,

(iii) $\omega(\lambda \delta) \leq(\lambda+1) \omega(\delta), \quad \lambda \geq 0$.

Further Leindler [3] has introduced the following metric on $H^{\omega}$ space:

$$
\|f\|_{\omega}=\|f\|_{c}+\sup _{x, y} \frac{|f(x)-f(y)|}{\omega(|x-y|)}, \quad x \neq y .
$$

In the case $\omega(\delta)=\delta^{\alpha}, 0<\alpha \leq 1$ the space $H^{\omega}$ reduces to $H_{\alpha}$ space (the norm $\|\cdot\|_{\omega}$ being replaced by $\|\cdot\|_{\alpha}$ ) which is introduced by Prössdorf [2]. It is known that [2]

$$
H_{\alpha} \subseteq H_{\beta} \subseteq \mathscr{C}_{2 \pi}, \quad 0 \leq \beta<\alpha \leq 1 .
$$

The degree of approximation problem in $H^{\omega}$ space has been studied by Leindler [3], Totik [4, 5], Mazhar and Totik [6], and Mazhar $[7,8]$. The space $H^{\omega}$ was further generalized by Das et al. [9] as follows.

For $f \in L_{p}[0,2 \pi], p \geq 1$, we write

$$
A(f, \omega)=\sup _{t \neq 0} \frac{\|f(\cdot+t)-f(\cdot)\|_{p}}{\omega(|t|)},
$$

where $\omega$ is a modulus of continuity. If

$$
\|f(\cdot+t)-f(\cdot)\|_{p}=O(\omega(|t|)),
$$

then we say that $f \in \operatorname{Lip}(\omega, p)$. We define

$$
\begin{aligned}
H_{p}^{(\omega)} & =\left\{f \in L_{p}[0,2 \pi], p \geq 1: A(f, \omega)<\infty\right\}, \\
\|f\|_{p}^{(\omega)} & =\|f\|_{p}+A(f, \omega) .
\end{aligned}
$$

It can be seen that $\|f\|_{p}^{(\omega)}$ is a norm in $H_{p}^{(\omega)}$. To prove the completeness of the space $H_{p}^{(\omega)}$ we use the completeness of $L_{p}(p \geq 1)$.

If we put $\omega(t)=t^{\alpha}, 0<\alpha \leq 1$, then $H_{p}^{(\omega)}$ reduces to $H(\alpha, p)$ space (with the norm $\|f\|_{p}^{(\omega)}$ replaced by $\|f\|_{(\alpha, p)}$ ) which is introduced earlier by Das et al. [10]. If $\omega(t) / t \rightarrow 0$ as $t \rightarrow 0$, then $f^{\prime}(x)$ exists and is 0 everywhere and $f$ is constant. Given the spaces $H_{p}^{(\omega)}$ and $H_{p}^{(v)}$, if $\omega(t) / v(t)$ is nondecreasing, then

$$
H_{p}^{(\omega)} \subseteq H_{p}^{(v)} \subseteq L_{p}, \quad p \geq 1,
$$

since

$$
\|f\|_{p}^{(v)} \leq \max \left(1, \frac{\omega(2 \pi)}{v(2 \pi)}\right)\|f\|_{p}^{(\omega)} .
$$

For $0 \leq \beta<\alpha \leq 1$, if we put $\omega(t)=t^{\alpha}$ and $v(t)=t^{\beta}$, then (12) reduces to the following:

$$
H_{(\alpha, p)} \subseteq H_{(\beta, p)} \subseteq L_{p}, \quad p \geq 1 .
$$

Note that the space $H_{(\alpha, \infty)}$ is the familiar $H_{\alpha}$ space introduced earlier by Prössdorf [2].
1.1. The Cesàro Transformation. Let $\sum_{n=0}^{\infty} u_{n}$ be an infinite series and let $\left(s_{n}\right)$ denote the sequence of its $n$th partial sums. Then the series $\sum u_{n}$ is said to be summable $(C, \mu)(\mu>-1)$ to the sum $s$ (finite), if (see [1], page 76)

$$
\sigma_{n}^{\mu}=\frac{S_{n}^{\mu}}{A_{n}^{\mu}} \longrightarrow s \quad \text { as } n \longrightarrow \infty,
$$

where $A_{n}^{\mu}$ and $S_{n}^{\mu}$ are defined by the following formulae:

$$
\begin{aligned}
\sum_{n=0}^{\infty} A_{n}^{\mu} x^{n}= & (1-x)^{-1-\mu} \\
\sum_{n=0}^{\infty} S_{n}^{\mu} x^{n} & =(1-x)^{-\mu} \sum_{n=0}^{\infty} s_{n} x^{n} \\
& =(1-x)^{-1-\mu} \sum_{n=0}^{\infty} u_{n} x^{n},
\end{aligned}
$$

where $(|x|<1), \mu>-1$. 77)

From the definition of $S_{n}^{\mu}$ and $A_{n}^{\mu}$ it follows that ([1], page

$$
\begin{aligned}
& S_{n}^{\mu}=\sum_{k=0}^{n} A_{n-k}^{\mu-1} s_{k}=\sum_{k=0}^{n} A_{n-k}^{\mu} u_{k} \\
& A_{n}^{\mu}=\left(\begin{array}{c}
n+\mu \\
\mu
\end{array}\right) \simeq \frac{n^{\mu}}{\Gamma(\mu+1)} \quad(\mu \neq-1,-2, \ldots) .
\end{aligned}
$$

The numbers $S_{n}^{\mu}$ and $\sigma_{n}^{\mu}$ are called, respectively, the Cesàro sums and the Cesàro means of order $\mu(\mu>-1)$ of the series $\sum u_{n}$. Applications of the Cesàro transformation can be found in engineering, for example, modal dynamics in earthquake engineering (see Chen and Hong [11], Chen et al. [12]).

1.2. Delayed Arithmetic Mean. Let $\sum_{n=0}^{\infty} u_{n}$ be an infinite series with sequence of arithmetic mean $\left\{\sigma_{n}\right\}$. The Delayed Arithmetic Mean $\sigma_{n, k}$ of $\sum u_{n}$ is given by ([1], page 80$)$

$$
\sigma_{n, k}=\left(1+\frac{n}{k}\right) \sigma_{n+k-1}-\frac{n}{k} \sigma_{n-1}
$$

where $k$ is a positive integer.

And it is known [1] that

$$
(C, 1) \subset \sigma_{n, k} \quad \text { if } \frac{n}{k}=O(1) \quad \text { as } k \longrightarrow \infty \text { with } n \text {. }
$$

For $k=n$,

$$
\sigma_{n, n}=2 \sigma_{2 n-1}-\sigma_{n-1},
$$

which may be called first type Delayed Arithmetic Mean.

But in this present paper we take $k=2 n$ for Delayed Arithmetic Mean, which is of the form

$$
\sigma_{n, 2 n}=\frac{1}{2}\left[3 \sigma_{3 n-1}-\sigma_{n-1}\right]
$$

This may be called second type Delayed Arithmetic Mean. 
Let $\sigma_{n}(f ; x)$ and $\sigma_{n, 2 n}(f ; x)$, respectively, denote the first arithmetic mean and second type Delayed Arithmetic Mean of (1). It is known that (see [1], page 88 and page 89) the Fejer's kernel

$$
\begin{gathered}
F_{n}(t)=\frac{2}{n+1}\left\{\frac{\sin \left(\frac{1}{2}\right)(n+1) t}{2 \sin \left(\frac{t}{2}\right)}\right\}^{2}, \\
\sigma_{n}(f ; x)=\frac{1}{\pi} \int_{0}^{\pi} F_{n}(t)\{f(x+t)+f(x-t)\} d t .
\end{gathered}
$$

It can be easily verified that

$$
\sigma_{n, 2 n}(f ; x)=\frac{1}{n \pi} \int_{0}^{\pi} \frac{\sin 2 n t \sin n t}{(2 \sin (t / 2))^{2}}\{f(x+t)+f(x-t)\} d t .
$$

We adopt the following additional notations:

$$
\begin{aligned}
G_{x, y}(t) & =\phi_{x+y}(t)-\phi_{x}(t), \\
l_{n}(x) & =\sigma_{n, 2 n}(f ; x)-f(x), \\
h & =h(n)=\frac{\pi}{n} .
\end{aligned}
$$

\section{Introduction}

Extensive investigations of approximation by polynomials $S_{n}$ and $\sigma_{n}$ involve mediocre approximation involving $O(\log n)$ times worse than the best approximation (see the remarks by [1], page 122). The following theorems, in particular, are being quoted to vindicate that $O(\log n)$ persists in the order of approximation involving $\sigma_{n}$ and $S_{n}$.

Das et al. [9] developed a new space $H_{p}^{(\omega)}$ and generalized the theorem of Leindler [3] to give the following.

Theorem A (see [9]). Let $v$ and $\omega$ be moduli of continuity such that $\omega / v$ is nondecreasing. If $f \in H_{p}^{(\omega)}, p \geq 1$, then

$$
\begin{aligned}
\left\|S_{n}(f ; x)-f(x)\right\|_{p}^{(v)}= & O\left(\frac{\omega(\pi / n)}{v(\pi / n)} \log n\right) \\
& +O(1) \frac{1}{n} \int_{\pi / n}^{\pi} \frac{\omega(t)}{t^{2} v(t)} d t .
\end{aligned}
$$

Theorem B (see [9]). If $f \in \operatorname{Lip}(\omega, p), p \geq 1$, and is of monotonic type, then

$$
\left\|S_{n}(f ; x)-f(x)\right\|_{p}=O(1) \frac{1}{n} \int_{1 / n}^{\pi} \frac{\omega(t)}{t^{2}} d t
$$

where $\omega$ is a modulus of continuity.

Recently Leindler [13] proved the following.

Theorem C ([13, Theorem 1]). Let $v$ and $\omega$ be moduli of continuity such that $\omega / v$ is nondecreasing; moreover, for some $0<\epsilon \leq 1$, let the function

$$
\gamma(t):=\gamma(v, \omega, \epsilon ; t):=t^{-\epsilon} \frac{\omega(t)}{v(t)}
$$

be nonincreasing. If $f \in H_{p}^{(\omega)}, p \geq 1$, then

$$
\left\|S_{n}-f\right\|_{p}^{(v)} \leq \frac{\omega(1 / n)}{v(1 / n)} \log n \quad \forall n
$$

Leindler [13] has derived Theorem $\mathrm{C}$ from Theorem A by making use of the fact that

$$
\int_{\pi / n}^{\pi} \frac{\omega(t)}{t^{2} v(t)} d t=O\left(\frac{\omega(\pi / n)}{v(\pi / n)} \log n\right)
$$

whenever $\omega(t) / t^{\epsilon} v(t) \quad(0<\epsilon \leq 1)$ is nonincreasing.

Quade [14] however proved the following.

Theorem D ([14, Theorem 5]). Let $f \in \operatorname{Lip}(\alpha, p), 0<\alpha \leq 1$; then

(i) $\left\|f-S_{n}(f, \cdot)\right\|_{p}=O\left(1 / n^{\alpha}\right), p>1$,

(ii) $\left\|f-S_{n}(f, \cdot)\right\|_{1}=O\left((\log n) / n^{\alpha}\right), p=1$.

Theorem E ([14, Theorem 6]). If $f \in \operatorname{Lip}(\alpha, p), 0<\alpha \leq 1$, then

(i) if $p>1$ or if $p=1, \alpha<1$,

$$
\left\|f-\sigma_{n}\right\|_{p}=O\left(\frac{1}{n^{\alpha}}\right)
$$

(ii) if $p=\alpha=1$,

$$
\left\|f-\sigma_{n}\right\|_{1}=O\left(\frac{\log n}{n}\right) .
$$

Prössdorf [2] obtained the following result concerning the degree of approximation of the Fourier series using Fejer's mean in the Hölder metric.

Theorem F (see [2]). Let $f \in H_{\alpha}(0<\alpha \leq 1)$ and $0 \leq \beta<$ $\alpha \leq 1$; then

$$
\left\|\sigma_{n}(f)-f\right\|_{\beta}=O(1) \begin{cases}\frac{1}{n^{\alpha-\beta}}, & 0<\alpha<1 \\ \frac{(1+\log n)^{1-\beta}}{n^{1-\beta}}, & \alpha=1 .\end{cases}
$$

The objective of the present paper is to establish theorems, which involve Jackson's order (see [15], page 56) not involving $O(\log n)$.

\section{Main Results}

We prove the following theorems.

Theorem 1. Let $v$ and $\omega$ be moduli of continuity such that $\omega / v$ is nondecreasing. If $f \in H_{p}^{(\omega)}, p \geq 1$, then

(I) $\left\|\sigma_{n, 2 n}(f ; \cdot)-f(\cdot)\right\|_{p}^{(v)}=O\left(\frac{1}{n}\right)+O(1) \frac{1}{n^{2}} \int_{\pi / n}^{\pi} \frac{\omega(t)}{t^{3} v(t)} d t$. 
If in addition $\omega(t) / t v(t)$ is nonincreasing, then

$$
\text { (II) }\left\|\sigma_{n, 2 n}(f ; \cdot)-f(\cdot)\right\|_{p}^{(v)}=O(1) \frac{1}{n^{2}} \int_{\pi / n}^{\pi} \frac{\omega(t)}{t^{3} v(t)} d t
$$

and a fortiori

$$
\text { (III) }\left\|\sigma_{n, 2 n}(f ; \cdot)-f(\cdot)\right\|_{p}^{(v)}=O\left(\frac{\omega(\pi / n)}{v(\pi / n)}\right) .
$$

Theorem 2. If $f \in \operatorname{Lip}(\omega, p), p \geq 1$, then

(I) $\left\|\sigma_{n, 2 n}(f ; \cdot)-f(\cdot)\right\|_{p}=O\left(\frac{1}{n}\right)+O(1) \frac{1}{n^{2}} \int_{\pi / n}^{\pi} \frac{\omega(t)}{t^{3}} d t$.

If in addition $\omega(t) / t$ is nonincreasing, then

$$
\text { (II) }\left\|\sigma_{n, 2 n}(f ; \cdot)-f(\cdot)\right\|_{p}=O(1) \frac{1}{n^{2}} \int_{\pi / n}^{\pi} \frac{\omega(t)}{t^{3}} d t
$$

and a fortiori

$$
\text { (III) }\left\|\sigma_{n, 2 n}(f ; \cdot)-f(\cdot)\right\|_{p}=O\left(\omega\left(\frac{\pi}{n}\right)\right) .
$$

We need the following lemma.

Lemma $3([9$, Lemma 1$])$. Let $v(t)$ and $\omega(t)$ be defined as in Theorem 1. Then for $0<t \leq \pi$ and $p \geq 1$

(i) $\|\phi .(t)\|_{p} \leq 2 \omega(t)$

(ii) $\left\|G_{\cdot, y}(t)\right\|_{p}=O(1)\left\{\begin{array}{c}\omega(t) \\ \omega(|y|),\end{array}\right.$

(iii) $\left\|G_{,, y}(t)\right\|_{p}=O(1) v(|y|)(\omega(t) / v(t))$,

(iv) $\left\|G_{,, y}(t)-G_{\cdot, y}(t+h)\right\|_{p}=O(1) v(|y|)(\omega(h) / v(h))$, where $h=\pi / n$.

\section{Proof of Theorem 1}

It may be easily verified that

$$
\frac{2}{n \pi} \int_{0}^{\pi} \frac{\sin 2 n t \sin n t}{(2 \sin (t / 2))^{2}} d t=1,
$$

and hence

$$
\begin{aligned}
l_{n}(x) & =\sigma_{n, 2 n}(f ; x)-f(x) \\
& =\frac{2}{n \pi} \int_{0}^{\pi} \phi_{x}(t) \frac{\sin 2 n t \sin n t}{(2 \sin (t / 2))^{2}} d t .
\end{aligned}
$$

By definition

$$
\left\|l_{n}\right\|_{p}^{(\omega)}=\left\|l_{n}\right\|_{p}+\sup _{y \neq 0} \frac{\left\|l_{n}(\cdot)-l_{n}(\cdot+y)\right\|_{p}}{\omega(|y|)} .
$$

Hence

$$
\begin{aligned}
& l_{n}(x+y)-l_{n}(x) \\
&=\frac{2}{n \pi} \int_{0}^{\pi}\left\{\phi_{x+y}(t)-\phi_{x}(t)\right\} \frac{\sin 2 n t \sin n t}{(2 \sin (t / 2))^{2}} d t \\
&=\frac{2}{n \pi} \int_{0}^{\pi}\left\{G_{x, y}(t)\right\} \frac{\sin 2 n t \sin n t}{(2 \sin (t / 2))^{2}} d t \\
&=\frac{2}{n \pi} \int_{0}^{h}\left\{G_{x, y}(t)\right\} \frac{\sin 2 n t \sin n t}{(2 \sin (t / 2))^{2}} d t \\
& \quad+\frac{2}{n \pi} \int_{h}^{\pi}\left\{G_{x, y}(t)\right\} \frac{\sin 2 n t \sin n t}{(2 \sin (t / 2))^{2}} d t \\
&= I+J .
\end{aligned}
$$

Since

$$
\frac{\sin 2 n t \sin n t}{(2 \sin (t / 2))^{2}}=O\left(n^{2}\right), \quad 0<t<\pi
$$

now, by the generalized Minkowski inequality and for $p \geq 1$, we have

$$
\begin{aligned}
\|I\|_{p} & \leq \frac{2}{n \pi} \int_{0}^{h}\left\|G_{\cdot, y}(t)\right\|_{p}\left|\frac{\sin 2 n t \sin n t}{(2 \sin (t / 2))^{2}}\right| d t \\
& =O(1) n v(|y|) \int_{0}^{h} \frac{\omega(t)}{v(t)} d t, \quad \text { (by Lemma 3) } \\
& =O(1) n v(|y|) h \frac{\omega(h)}{v(h)} \\
& =O(1) v(|y|) \frac{\omega(h)}{v(h)}
\end{aligned}
$$

Now,

$$
\begin{aligned}
J= & \frac{2}{n \pi} \int_{h}^{\pi} G_{x, y}(t) \frac{\sin 2 n t \sin n t}{(2 \sin (t / 2))^{2}} d t \\
= & \frac{2}{n \pi} \int_{h}^{\pi} G_{x, y}(t) \sin 2 n t \sin n t\left\{\frac{1}{(2 \sin (t / 2))^{2}}-\frac{1}{t^{2}}\right\} d t \\
& +\frac{2}{n \pi} \int_{h}^{\pi} G_{x, y}(t) \frac{\sin 2 n t \sin n t}{t^{2}} d t \\
= & J_{1}+J_{2} .
\end{aligned}
$$

Since $\left\{1 /(2 \sin (t / 2))^{2}-1 / t^{2}\right\}$ is bounded and by Lemma 3 , we get

$$
\begin{aligned}
\left\|J_{1}\right\|_{p} & \leq \frac{2}{n \pi} \int_{h}^{\pi}\left\|G_{\cdot, y}(t)\right\|_{p} d t \\
& =O(1) \frac{1}{n} v(|y|) \int_{h}^{\pi} \frac{\omega(t)}{v(t)} d t \\
& =O(1) \frac{1}{n} v(|y|) .
\end{aligned}
$$


Now,

$$
J_{2}=\frac{2}{n \pi} \int_{h}^{\pi} G_{x, y}(t) \frac{\sin 2 n t \sin n t}{t^{2}} d t
$$

Replacing $t$ with $t+h$ in (48), we obtain

$$
J_{2}=-\frac{2}{n \pi} \int_{0}^{\pi-h} G_{x, y}(t+h) \frac{\sin 2 n t \sin n t}{(t+h)^{2}} d t .
$$

From (48) and (49), it follows that

$$
\begin{aligned}
J_{2}= & \frac{1}{n \pi} \int_{h}^{\pi} G_{x, y}(t) \frac{\sin 2 n t \sin n t}{t^{2}} d t \\
& -\frac{1}{n \pi} \int_{0}^{\pi-h} G_{x, y}(t+h) \frac{\sin 2 n t \sin n t}{(t+h)^{2}} d t \\
= & \frac{1}{n \pi} \int_{h}^{\pi} G_{x, y}(t) \frac{\sin 2 n t \sin n t}{t^{2}} d t \\
& -\frac{1}{n \pi} \int_{0}^{h} G_{x, y}(t+h) \frac{\sin 2 n t \sin n t}{(t+h)^{2}} d t \\
& -\frac{1}{n \pi} \int_{h}^{\pi} G_{x, y}(t+h) \frac{\sin 2 n t \sin n t}{(t+h)^{2}} d t \\
& +\frac{1}{n \pi} \int_{\pi-h}^{\pi} G_{x, y}(t+h) \frac{\sin 2 n t \sin n t}{(t+h)^{2}} d t \\
= & \frac{1}{n \pi} \int_{h}^{\pi}\left\{\frac{G_{x, y}(t)}{t^{2}}-\frac{G_{x, y}(t+h)}{(t+h)^{2}}\right\} \sin 2 n t \sin n t d t \\
& +\frac{1}{n \pi} \int_{\pi-h}^{h} G_{x, y}(t+h) \frac{\sin 2 n t \sin n t}{(t+h)^{2}} d t \\
& K-G_{x, y}(t+h) \frac{\sin 2 n t \sin n t}{(t+h)^{2}} d t
\end{aligned}
$$

Since $\sin 2 n t \sin n t=O\left(n^{2} t^{2}\right), 0<t<\pi$, it follows that

$$
\begin{aligned}
\|L\|_{p} & \leq \frac{1}{n \pi} \int_{0}^{h}\left\|G_{\cdot, y}(t+h)\right\|_{p} \frac{n^{2} t^{2}}{(t+h)^{2}} d t \\
& =O(1) \frac{1}{n} v(|y|) \int_{0}^{h} \frac{\omega(t+h)}{v(t+h)} n^{2} d t \\
& =O(1) n v(|y|) \int_{0}^{h} \frac{\omega(t+h)}{v(t+h)} d t \\
& =O(1) n v(|y|) \frac{\omega(2 h)}{v(2 h)} \int_{0}^{h} d t \\
& =O(1) n v(|y|) \frac{\omega(h)}{v(h)} h \\
& =O(1) v(|y|) \frac{\omega(h)}{v(h)}
\end{aligned}
$$

as $\omega(2 h) \leq 3 \omega(h)$ and $v(2 h) \geq v(h)$.
Since $|\sin 2 n t \sin n t| \leq 1$, for $t \in(\pi-h, \pi)$, it follows that

$$
\begin{aligned}
\|M\|_{p} & \leq \frac{1}{n \pi} \int_{\pi-h}^{\pi}\left\|G_{\cdot, y}(t+h)\right\|_{p} \frac{d t}{(t+h)^{2}} \\
& =O(1) \frac{1}{n} v(|y|) \int_{\pi-h}^{\pi} \frac{\omega(t+h)}{v(t+h)} \frac{d t}{(t+h)^{2}} \\
& =O(1) \frac{1}{n} v(|y|) \int_{\pi}^{\pi+h} \frac{\omega(\theta)}{v(\theta)} \frac{d \theta}{\theta^{2}} \\
& =O(1) \frac{1}{n^{2}} v(|y|) .
\end{aligned}
$$

Now,

$$
\begin{aligned}
K=\frac{1}{n \pi}\left\{\int_{h}^{\pi} G_{x, y}(t) \frac{\sin 2 n t \sin n t}{t^{2}} d t\right. \\
\quad-\int_{h}^{\pi} G_{x, y}(t+h) \frac{\sin 2 n t \sin n t}{t^{2}} d t \\
\quad+\int_{h}^{\pi} G_{x, y}(t+h) \frac{\sin 2 n t \sin n t}{t^{2}} d t \\
\left.\quad-\int_{h}^{\pi} G_{x, y}(t+h) \frac{\sin 2 n t \sin n t}{(t+h)^{2}} d t\right\} \\
=\frac{1}{n \pi} \int_{h}^{\pi}\left\{G_{x, y}(t)-G_{x, y}(t+h)\right\} \frac{\sin 2 n t \sin n t}{t^{2}} d t \\
+\frac{1}{n \pi} \int_{h}^{\pi} G_{x, y}(t+h) \sin 2 n t \sin n t\left\{\frac{1}{t^{2}}-\frac{1}{(t+h)^{2}}\right\} d t \\
=K_{1}+K_{2} .
\end{aligned}
$$

Since $|\sin 2 n t \sin n t| \leq 1$, for $t \in(h, \pi)$, using Lemma 3 (iv), we get

$$
\begin{aligned}
\left\|K_{1}\right\|_{p} & \leq \frac{1}{n \pi} \int_{h}^{\pi}\left\|G_{\cdot, y}(t)-G_{\cdot, y}(t+h)\right\|_{p} \frac{d t}{t^{2}} \\
& =O(1) \frac{1}{n} v(|y|) \frac{\omega(h)}{v(h)} \int_{h}^{\pi} \frac{d t}{t^{2}} \\
& =O(1) v(|y|) \frac{\omega(h)}{v(h)} .
\end{aligned}
$$

Similarly,

$$
\begin{aligned}
\left\|K_{2}\right\|_{p} & \leq \frac{1}{n \pi} \int_{h}^{\pi}\left\|G_{,, y}(t+h)\right\|_{p}\left\{\frac{1}{t^{2}}-\frac{1}{(t+h)^{2}}\right\} d t \\
& =O(1) \frac{1}{n} v(|y|) \int_{h}^{\pi} \frac{\omega(t+h)}{v(t+h)} \frac{(t+h)^{2}-t^{2}}{t^{2}(t+h)^{2}} d t \\
& =O(1) \frac{1}{n^{2}} v(|y|) \int_{h}^{\pi} \frac{\omega(t+h)}{v(t+h)} \frac{d t}{t^{3}} \\
& =O(1) \frac{1}{n^{2}} v(|y|) \int_{h}^{\pi} \frac{\omega(t)}{v(t)} \frac{d t}{t^{3}} .
\end{aligned}
$$


Collecting the results from (45)-(55), we obtain

$$
\sup _{y \neq 0} \frac{\left\|l_{n}(\cdot)-l_{n}(\cdot+y)\right\|_{p}}{v(|y|)}=O\left(\frac{1}{n}\right)+O(1) \frac{1}{n^{2}} \int_{\pi / n}^{\pi} \frac{\omega(t)}{t^{3} v(t)} d t
$$

Proceeding in the similar lines, it can be proved that

$$
\left\|l_{n}\right\|_{p}=O\left(\frac{1}{n}\right)+O(1) \frac{1}{n^{2}} \int_{\pi / n}^{\pi} \frac{\omega(t)}{t^{3}} d t .
$$

Hence collecting results of (42), (56), and (57), we obtain

$$
\left\|l_{n}\right\|_{p}^{(v)}=O\left(\frac{1}{n}\right)+O(1) \frac{1}{n^{2}} \int_{\pi / n}^{\pi} \frac{\omega(t)}{t^{3} v(t)} d t
$$

which ensures (I) of Theorem 1 .

If in addition $\omega(t) / t v(t)$ is nonincreasing, we have

$$
\frac{1}{n^{2}} \int_{\pi / n}^{\pi} \frac{\omega(t)}{t^{3} v(t)} d t \geq \frac{1}{n^{2}} \frac{\omega(\pi)}{\pi v(\pi)} \int_{\pi / n}^{\pi} \frac{d t}{t^{2}} \geq \frac{C}{n},
$$

where $C$ is some positive constant. Further

$$
\frac{1}{n^{2}} \int_{\pi / n}^{\pi} \frac{\omega(t)}{t^{3} v(t)} d t \leq \frac{1}{n^{2}} \frac{n}{\pi} \frac{\omega(\pi / n)}{v(\pi / n)} \int_{\pi / n}^{\pi} \frac{d t}{t^{2}}=O\left(\frac{\omega(\pi / n)}{v(\pi / n)}\right) .
$$

Using (59) and (60) in (58), we get (II) and (III) of Theorem 1. This completes the proof.

\section{Proof of Theorem 2}

We adopt the technique used in the proof of Theorem 1 and use the fact that $\|\phi .(t)\|_{p}=O(\omega(t))$ whenever $f \in$ $\operatorname{Lip}(\omega, p), p \geq 1$, to prove Theorem 2 .

\section{Remarks and Corollaries}

Comparing Theorem 1 with Theorem A and Theorem $\mathrm{C}$ we observe that trigonometric polynomial $\sigma_{n, 2 n}(f ; x)$ provides sharper estimation than those obtained by sequence of partial sums of a Fourier series. The comparison of Theorem 2 with Theorem B also exhibits the advantage of $\sigma_{n, 2 n}(f ; x)$ over the sequence of partial sums of the Fourier series in the degree of approximation problem.

We specialize $\omega(t)$ and $v(t)$ to obtain the following corollaries from Theorems 1 and 2. Taking $\omega(t)=t^{\alpha}$ and $v(t)=t^{\beta}, 0 \leq \beta<\alpha \leq 1$ in Theorem 1 , we get the following.

Corollary 4. If $f \in H(\alpha, p), p \geq 1,0 \leq \beta<\alpha \leq 1$, then

$$
\left\|\sigma_{n, 2 n}(f ; \cdot)-f(\cdot)\right\|_{(\beta, p)}=O\left(\frac{1}{n^{\alpha-\beta}}\right) .
$$

Taking $p=\infty$ in Corollary 4 , we obtain the following.

Corollary 5. If $f \in H_{\alpha}, 0 \leq \beta<\alpha \leq 1$, then

$$
\left\|\sigma_{n, 2 n}(f ; \cdot)-f(\cdot)\right\|_{\beta}=O\left(\frac{1}{n^{\alpha-\beta}}\right) .
$$

The above corollary can be compared with Theorem F.

Taking $\omega(t)=t^{\alpha}, 0<\alpha \leq 1$ in Theorem 2 , we obtain the following.

Corollary 6. If $f \in \operatorname{Lip}(\alpha, p), 0<\alpha \leq 1$ and $p \geq 1$, then

$$
\left\|\sigma_{n, 2 n}(f ; \cdot)-f(\cdot)\right\|_{p}=O\left(\frac{1}{n^{\alpha}}\right) .
$$

For the space $\operatorname{Lip}(1,1)$ Quade has provided the result (32) of Theorem E using $\sigma_{n}(f ; x)$ trigonometric polynomial. Estimate given in (32) certainly is not of Jackson's order. Therefore in Corollary 6 a more general trigonometric polynomial (namely, $\sigma_{n, 2 n}(f ; x)$ ) has been taken to ensure Jackson's order, which is sharper than the one given in (32).

\section{Conflict of Interests}

The authors declare that there is no conflict of interests regarding the publication of this paper.

\section{Acknowledgment}

The authors are grateful to the referee for his valuable suggestions and criticism which led to the improvement of the paper.

\section{References}

[1] A. Zygmund, Trigonometric Series, vol. 1-2, Cambridge University Press, New York, NY, USA, 2nd edition, 1993.

[2] S. Prössdorf, "Zur Konvergenz der Fourierreihen hölderstetiger Funktionen," Mathematische Nachrichten, vol. 69, pp. 7-14, 1975.

[3] L. Leindler, "Generalizations of Prössdorf's theorems," Consilium Instituti Mathematici Academiae Scientiarum Hungaricae. Studia Scientiarum Mathematicarum Hungarica, vol. 14, no. 4, pp. 431-439, 1979.

[4] V. Totik, "On the strong approximation of Fourier series," Acta Mathematica Academiae Scientiarum Hungaricae, vol. 35, no. 12, pp. 151-172, 1980.

[5] V. Totik, "Strong approximation by the de la Vallée-Poussin and Abel means of Fourier series," The Journal of the Indian Mathematical Society, vol. 45, no. 1-4, pp. 85-108, 1981.

[6] S. M. Mazhar and V. Totik, "Approximation of continuous functions by $T$-means of Fourier series," Journal of Approximation Theory, vol. 60, no. 2, pp. 174-182, 1990.

[7] S. M. Mazhar, "Approximation by the partial sums of Fourier series," International Mathematical Journal of Analysis and its Applications, vol. 11, no. 2-3, pp. 149-154, 1991.

[8] S. M. Mazhar, "Approximation by logarithmic means of a Fourier series," in Approximation Theory VI, C. K. Chui, L. L. Schumaker, and J. D. Wards, Eds., vol. 2, pp. 421-424, Academic Press, Boston, Mass, USA, 1989.

[9] G. Das, A. Nath, and B. K. Ray, "An estimate of the rate of convergence of Fourier series in the generalized Hölder metric," in Analysis and Applications, pp. 43-60, Narosa, New Delhi, India, 2002.

[10] G. Das, T. Ghosh, and B. K. Ray, "Degree of approximation of functions by their Fourier series in the generalized Hölder metric," Indian Academy of Sciences. Proceedings. Mathematical Sciences, vol. 106, no. 2, pp. 139-153, 1996. 
[11] J. T. Chen and H. K. Hong, "Review of dual boundary element methods with emphasis on hypersingular integrals and divergent series," Applied Mechanics Reviews, vol. 52, no. 1, pp. 17-33, 1999.

[12] J. T. Chen, H. K. Hong, C. S. Yeh, and S. W. Chyuan, "Integral representations and regularizations for a divergent series solution of a beam subjected to support motions," Earthquake Engineering and Structural Dynamics, vol. 25, no. 9, pp. 909925, 1996.

[13] L. Leindler, "A relaxed estimate of the degree of approximation by Fourier series in generalized Hölder metric," Analysis Mathematica, vol. 35, no. 1, pp. 51-60, 2009.

[14] E. S. Quade, “Trigonometric approximation in the mean," Duke Mathematical Journal, vol. 3, no. 3, pp. 529-543, 1937.

[15] G. G. Lorentz, Approximation of Functions, Syracuse University, 1966. 


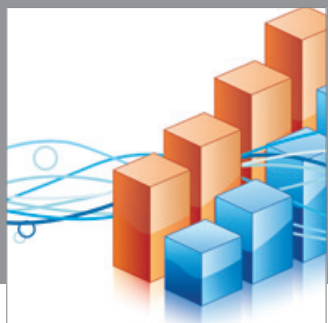

Advances in

Operations Research

mansans

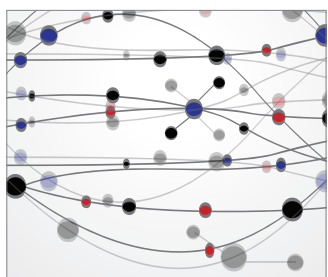

The Scientific World Journal
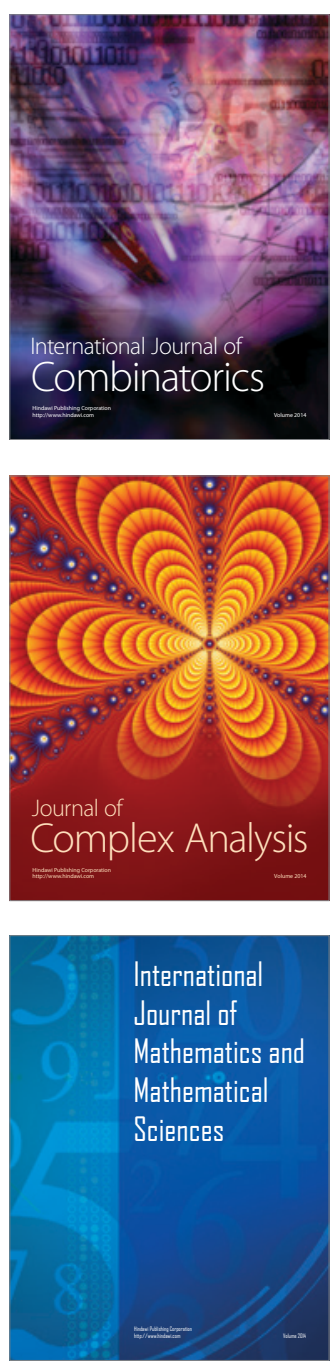
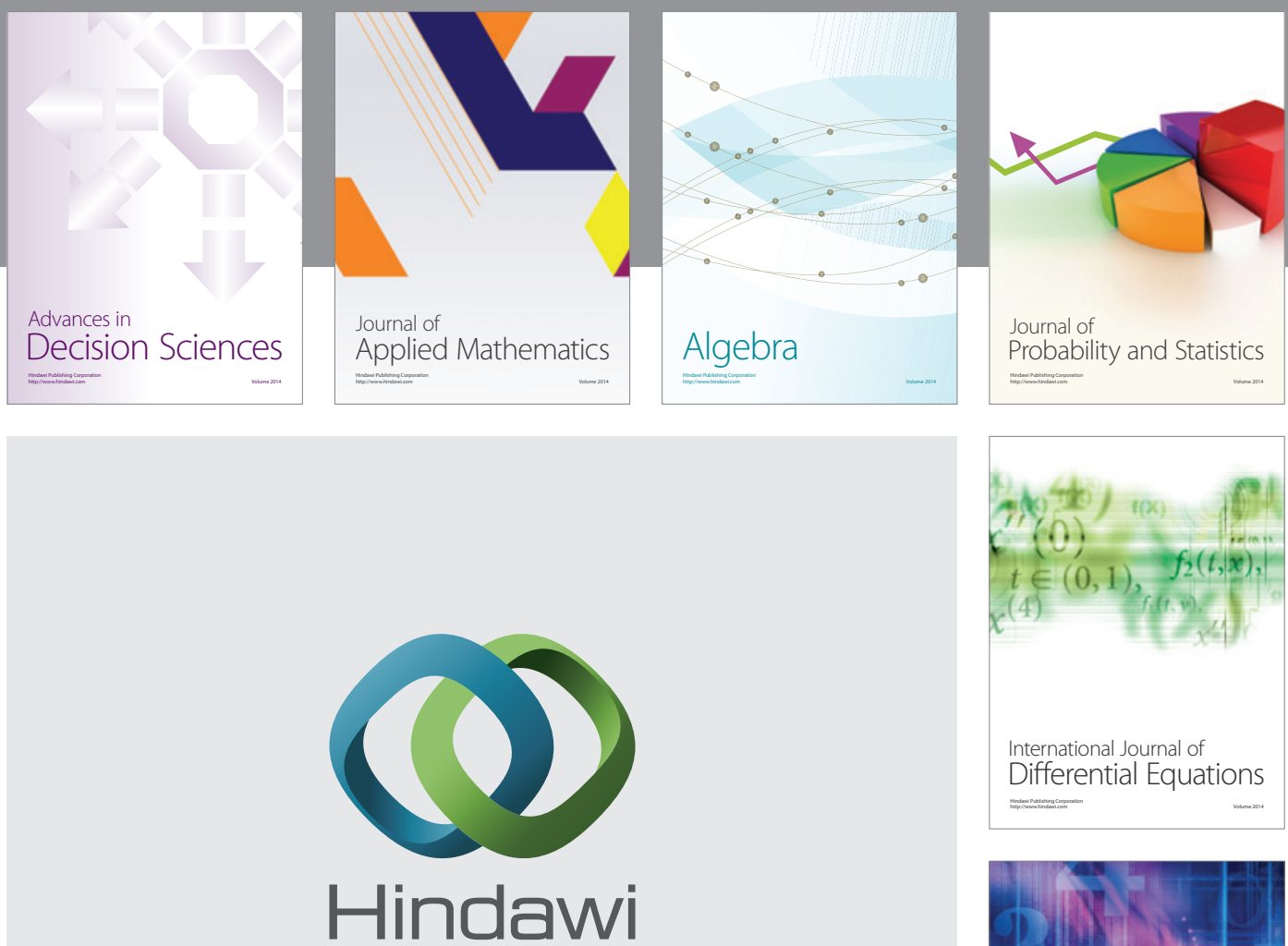

Submit your manuscripts at http://www.hindawi.com
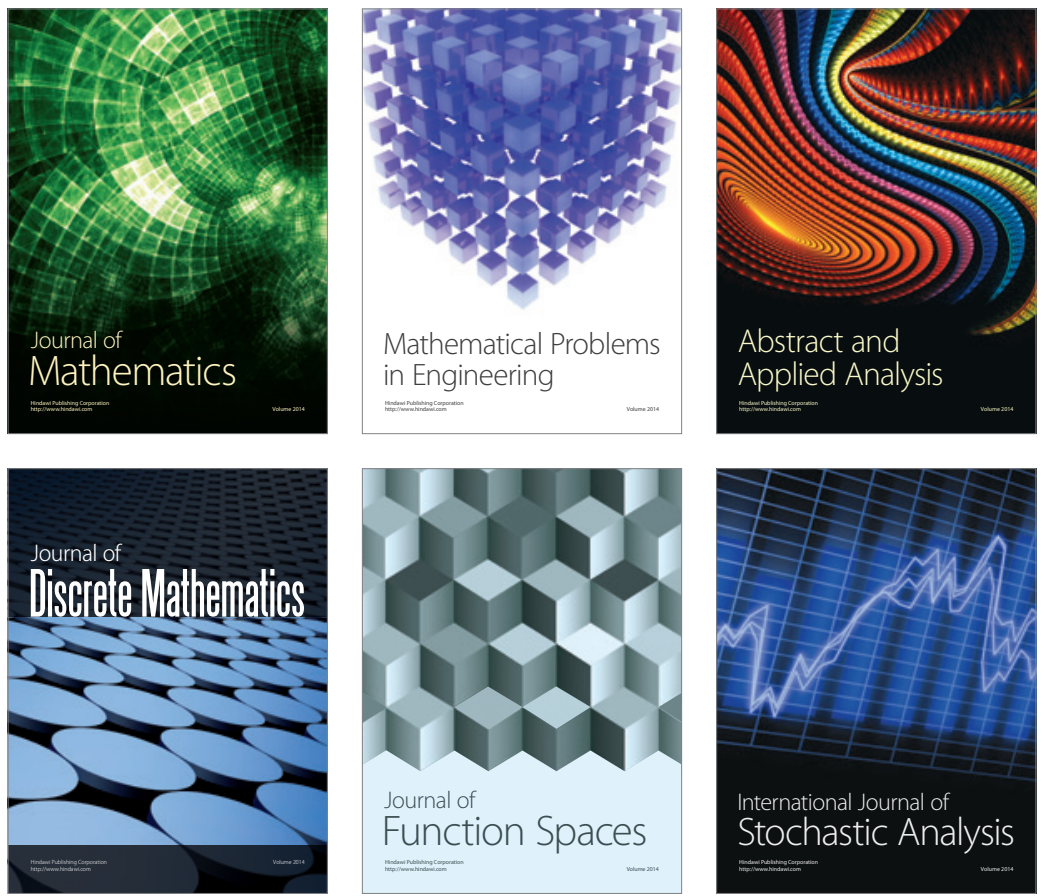

Journal of

Function Spaces

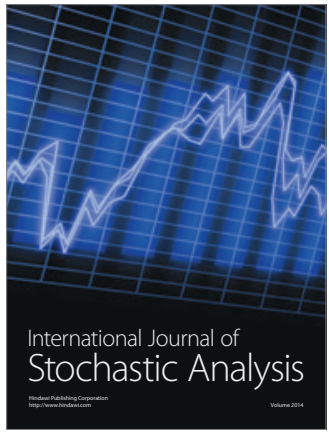

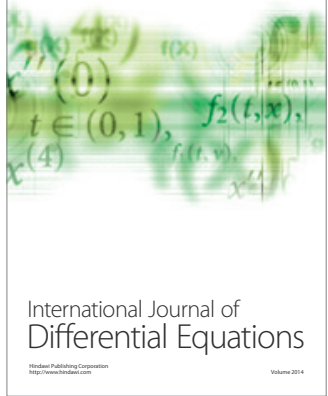
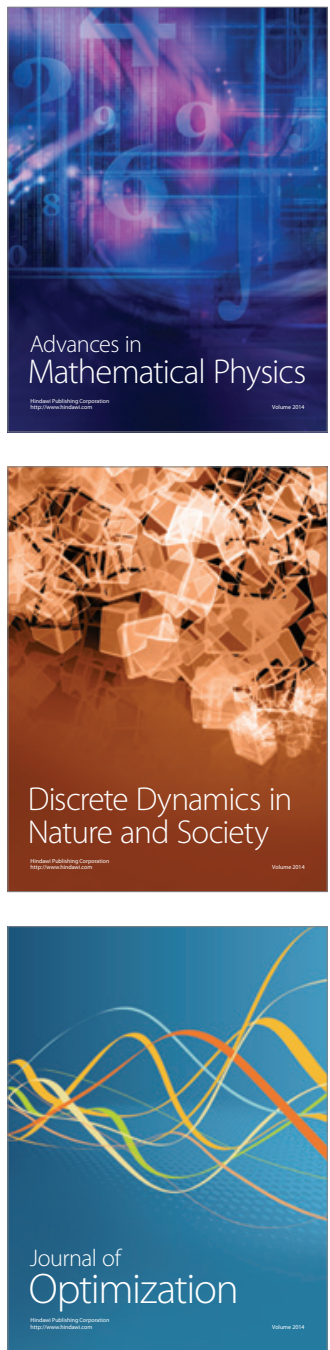